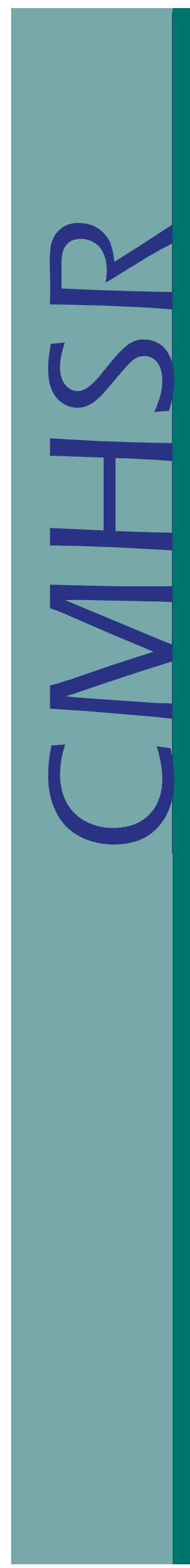

February 2006

Vol 3, Issue 2
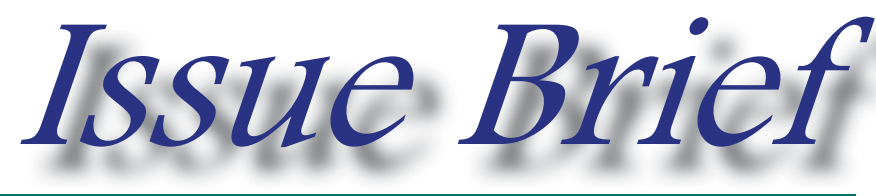

\title{
Restraint and Seclusion: Can They Become Obsolete Practices?
}

Goran Jovanovic, B.S., Matthew Johnsen, Ph.D.

$\mathrm{F}$ acilities that treat individuals with mental illness strive to offer safe environments which are conducive to treatment and foster human potential. Environments that utilize restraint and seclusion $(\mathrm{R} / \mathrm{S})$ as a treatment option may engender fear, and for individuals who have histories of traumatic victimization, can trigger a recapitulation of traumatic experiences thereby exacerbating symptoms of Post-Traumatic Stress Disorder (PTSD) or other mental illness. ${ }^{1}$ These types of flashback episodes can be counterproductive to treatment.

Incidents which lead to $\mathrm{R} / \mathrm{S}$ often involve violent and harmful encounters between patients and staff, eliciting distress in both. Many patients in psychiatric settings report that $\mathrm{R} / \mathrm{S}$ is among the most harmful and traumatic events that they have experienced. ${ }^{1}$ Using $\mathrm{R} / \mathrm{S}$ is controversial, and has ethical implications. In 1998, the Hartford Courant's series on deaths associated with R/S reported 142 deaths in the United States from these techniques from 1988 to $1998 .^{2}$

Psychiatric hospitals have been using R/S for centuries to control people with disturbed or violent behaviors. ${ }^{3}$ Many facilities use R/S as a "measure of last resort." The International Society of Psychiatric-Mental Health Care Nurses (ISPN) indicates that R/S is "an emergency clinical intervention employed only as a last effort when less restrictive alternatives have failed to ensure safety for patients, staff and families." 4 Restraint is any method, physical or chemical, that restricts one's freedom of movement or access to one's body. Seclusion is the process of confining an individual to a room and physically preventing them from leaving for any period of time.

C 2005 Center for Mental Health Services Research Department of Psychiatry University of Massachusetts Medical School

\section{Efforts to Reduce or Eliminate R/S}

Proponents of $\mathrm{R} / \mathrm{S}$ reduction initiatives have stated that the most important aspect of these efforts is culture change.3,5 SAMHSA Administrator Charles G. Curie, suggests that "success begins with a change in culture, from one of power to empowerment, from coercion, to caring and from hopelessness to hope." To initiate a culture change "leadership at the top is essential..." In addition, he suggests that efforts to reduce R/S may benefit from staff training, better data collection and dissemination and resolving to use R/S only when "the potential exists for imminent physical danger to the patient or others." 5

Some administrators and researchers see $\mathrm{R} / \mathrm{S}$ as an indication of treatment failure, ${ }^{5}$ challenging mental health programs to find better ways to deal with crises. Methods include training staff in more effective de-escalation techniques, introduction of Psychiatric Emergency Response Teams (PERTs) as well as increasing staff-to-patient ratios. ${ }^{3}$ Others have suggested adopting a "Best Practice Model" for successfully reducing R/S.6 "Best Practice" is defined as collecting and managing information and resources in a cost effective manner. ${ }^{6}$

\section{Studies of R/S Reduction}

I. One long-term study examined patterns of use of R/S from 1990 to 2000 in Pennsylvania's state hospital system. ${ }^{3}$ Patterns over the 11 -year period among nine sites included average decreases in:

- Rates of seclusion from 7.2/1000 to $0.3 / 1000$ patient days - a reduction of $96 \%$

- Duration of seclusion from 11.6 to 1.3 hours - a reduction of $89 \%$

- Rates of restraint from 6.4 episodes/1000 days to 1.2 episodes/1000 days - a reduction of $81 \%$ 
- Duration of restraint from 12.1 to 1.9 hours - a reduction of $84 \%$

The authors attributed these reductions to a number of factors including effective leadership, state policy change, the implementation of PERTs and an increased staff-topatient ratio on hospital units. ${ }^{3}$

II. A retrospective analysis of a public psychiatric hospital's attempts to reduce $\mathrm{R} / \mathrm{S}$ evaluated and reviewed a variety of interventions that were successful in reducing R/S.7

The results of this study showed that the use of $\mathrm{R} / \mathrm{S}$ decreased $75 \%$ over a 5 year period. The only variable that was significantly associated with reduced use of $\mathrm{R} / \mathrm{S}$ was a changed process for identifying critical cases and initiating a clinical and administrative case review. This change was a reduction in the number of restraint or seclusion applications permitted on the patient before their case was labeled as critical. Critical cases required administrative and clinical review.

This study underscores the importance of clinical and administrative priorities in efforts to reduce R/S. ${ }^{6}$ The findings support the belief that leadership is the most important priority in any attempts to change the culture and consequently reduce the use of R/S. $3,5,7$

III. One Massachusetts study compared the difference between costs of restraint usage one year prior, and one year after, a reduction initiative. ${ }^{8}$

- $\quad$ Facility wide, restraint use decreased from 3991 to 373 episodes after implementation - a reduction of $91 \%$

- This reduction was associated with a reduction in facility costs associated with the application of restraints from $\$ 1,446,740$ to $\$ 117,036$ - a reduction of $92 \%$.

In addition, the reduction of restraints was associated with better patient treatment outcomes, more effective usage of staff time, and decrease in the use of sick time and staff turnover. ${ }^{8}$

\section{Future Directions}

Creating treatment environments where $\mathrm{R} / \mathrm{S}$ are practices of the past will be challenging. When used conscientiously, and only as a last resort, some argue that it can keep individuals safe from harm; however, others argue that $\mathrm{R} / \mathrm{S}$ can create an environment of tension and fear. Can treatment environments be safe, conducive to treatment and foster human potential if they use $\mathrm{R} / \mathrm{S}$ as a treatment modality?

Massachusetts is one of eight states that have recently been awarded funding by SAMHSA in order to carry out evaluations of $\mathrm{R} / \mathrm{S}$ reduction strategies implemented by facilities. Currently, CMHSR, in collaboration with the Massachusetts Department of Mental Health, is involved in the evaluation of eleven Massachusetts sites. These efforts promise to identify successful strategies for reducing $\mathrm{R} / \mathrm{S}$, and may provide examples of psychiatric settings that have successfully eliminated them.

\section{References}

1. Frueh, B.C., Knapp, R.G., Cusack, K.J., Grubaugh, A.L., Sauvegeot, J.A., Cousins, V.C. (2005). Patients' reports of traumatic or harmful experiences within a psychiatric setting. Psychiatric Services, 56, 1123-1133.

2. Weiss E.M. 1998. Deadly restraint: A Hartford Courant investigative report. Retrieved November 25, 2005 from http://www.charlydmiller.com/LIB05/ 1998hartforddata.html.

3. Smith, G.M., Davis, R.H., Bixler, E.O., Lin, H.M., Altenor, A., Altenor, R.J. et al. (2005). Pennsylvania state hospital system's seclusion and restraint reduction program. Psychiatric Services, 56,1115-1122.

4. International Society of Psychiatric-Mental Health Nurses. (1999, November). The Use of Seclusion and Restraint. Retrieved December 1, 2005 from http://www. ispn-psych.org/docs/99Restraint-Seclusion.pdf

5. Curie, C.G. (2005). SAMHSA's commitment to eliminating the use of seclusion and restraint. Psychiatric Services, 56, 1139-1140.

6. Visalli, H. McNasser, G. (1997). Striving towards a best practice model for a restraint-free environment. Journal of Nursing Care Quality, 6, 1-4.

7. Donat, D.C. (2003). An analysis of successful efforts to reduce the use of seclusion and restraint at a public psychiatric hospital. Psychiatric Services, 54, 1119-1123.

8. LeBel, J., Goldstein, R. (2005). The Economic cost of using restraint and the value added by restraint reduction or elimination. Psychiatric Services, 56, 1109-1114.

Visit us on-line at www.umassmed.edu/cmhsr 